\title{
Possible Hydromagnetic Simulation of Cosmical Phenomena in the Laboratory
}

\author{
Winston H. Bostick* \\ Stevens Institute of Technology, Hoboken, New Jersey
}

\begin{abstract}
A TECHNIQUE has been developed ${ }^{1-3}$ whereby ionized gas (plasma) can be projected, by magnetic forces, at speeds of $3 \times 10^{7} \mathrm{~cm} / \mathrm{sec}$ through a vacuum region free from a magnetic field. The plasma has also been projected across magnetic fields in vacuum at speeds of $10^{7} \mathrm{~cm} / \mathrm{sec}$. It is further possible to have present in the magnetic field a background electrically conducting medium in the form of a low-pressure (about one micron) ionized gas. This gas is photoionized by the ultraviolet light from the plasma gun. With the background conducting medium we can calculate, following Spitzer, ${ }^{4}$ the electric resistivity $\eta$ transverse to a strong magnetic field to be $\eta=1.29 \times 10^{13}(Z \ln \Lambda) T^{-\frac{3}{2}} \mathrm{emu}$ $=10^{6} \mathrm{emu}$ for $T=10^{5}{ }^{\circ} \mathrm{K}$ (electron temperature), $Z=1$, $\ln \Lambda=2.3$. The magnetic Reynolds number $R_{m}$ for $L=10$ $\mathrm{cm}$ and $V=10^{7} \mathrm{~cm} / \mathrm{sec}$ is thus $R_{m}=4 \pi L V / \eta=10^{3}$. Consequently, there is no doubt that with this technique we are producing magnetohydrodynamic phenomena in the laboratory.

We might ask whether our laboratory experiments satisfy the similarity transformations outlined by Alfven. ${ }^{5}$ The length transformation ratio is $10^{22}$ in passing from the diameter of the galaxy $\left(10^{23} \mathrm{~cm}\right)$ to the diameter $(10 \mathrm{~cm})$ of our laboratory apparatus. The transformation ratios, both desired and actual, for the other quantities involved are given in Table I. Thus, the time $T$ and velocity $v$ transform correctly in the laboratory
\end{abstract}

experiments. Although the electrical conductivity $\sigma$ has not been transformed according to the rules, it should be possible nevertheless to simulate cosmical magnetohydrodynamics as long as the laboratory magnetic Reynolds number $R_{m}$ is large. Although the ion density $n$ also does not transform according to the rules, we believe that no serious defect in the simulation of the phenomena should result. We need only have the ion density high enough so that the phenomena can be made visible by the recombination light of the ions and electrons. Gravitational effects in the galaxy will have to be simulated by inertial forces in the laboratory.

There is a serious question concerning the way in which the magnetic field transforms, since its transformation ratio differs by a factor of $10^{12}$. However, this situation is not hopeless because the drift velocities of the individual ions and electrons in the plasma across the magnetic field are given by

$$
\mathbf{V}_{D}=\frac{c \mathbf{H} \times}{e H^{2}}\left[e \mathbf{E}+m \frac{d \mathbf{V}_{D}}{d t}+\mu \nabla H+\frac{1}{n} \nabla n k T\right]
$$

Although there is a net value of $H$ to the first power in the denominator, the drift velocity $\mathbf{V}_{D}$ of the plasma as a whole scales properly. Therefore, the various force terms in the numerator must scale with approximately the same incorrect factor as $H$ does, in order that the

TABLE I. Similarity transformations between the galaxy and the laboratory.

\begin{tabular}{|c|c|c|c|c|}
\hline $\begin{array}{l}\text { Galaxy } \\
\text { Laboratory } \\
\text { Actual ratio }\end{array}$ & $\begin{array}{l}\text { Length } L \\
10^{23} \mathrm{~cm} \\
10 \mathrm{~cm} \\
10^{22}\end{array}$ & $\begin{array}{l}\quad \text { Time } T \\
3 \times 10^{17} \mathrm{sec} \\
3 \times 10^{-5} \mathrm{sec} \\
10^{22}\end{array}$ & & $\begin{array}{c}\text { Ideal transformation ratio } \\
\qquad\left\{(\text { ratio })^{1}=10^{22}\right.\end{array}$ \\
\hline $\begin{array}{l}\text { Galaxy } \\
\text { Laboratory } \\
\text { Actual ratio }\end{array}$ & $\begin{array}{l}\text { Particle } \\
\text { energy }\end{array}$ & $\begin{array}{l}\quad \text { Velocity } \\
10^{7} \mathrm{~cm} / \mathrm{sec} \\
10^{6}-10^{7} \mathrm{~cm} / \mathrm{sec} \\
\sim 1\end{array}$ & Potential current resistance & $(\text { ratio })^{0}=1$ \\
\hline $\begin{array}{l}\text { Galaxy } \\
\text { Laboratory } \\
\text { Actual ratio }\end{array}$ & $\begin{array}{l}\text { Electric } \\
\text { field } E\end{array}$ & $\begin{array}{l}\text { Magnetic } \\
\quad \text { field } H \\
10^{-6} \text { gauss } \\
6 \times 10^{3} \\
1.6 \times 10^{-10}\end{array}$ & $\begin{array}{l}\text { Conductivity, ion density, } \\
\qquad \begin{array}{c}1 \mathrm{~cm}^{-3} \\
10^{16} \\
10^{-16}\end{array}\end{array}$ & $(\text { ratio })^{-1}=10^{-22}$ \\
\hline
\end{tabular}

* The experimental work described here was performed at the University of California Radiation Laboratory at Livermore, and was sponsored by the U. S. Atomic Energy Commission.

1 W. H. Bostick, Phys. Rev. 104, 292 (1956).

2 W. H. Bostick and O. A. Twite, Nature 179, 214 (1957).

3 W. H. Bostick, Phys. Rev. 106, 404 (1957).

${ }^{4} \mathrm{~L}$. Spitzer, Physics of Fully Ionized Gases (Interscience Publishers, Inc., New York, 1956), p. 86

5 H. Alfven, Cosmical Electrodynamics (Oxford University Press, New York, 1950), p. 39. 


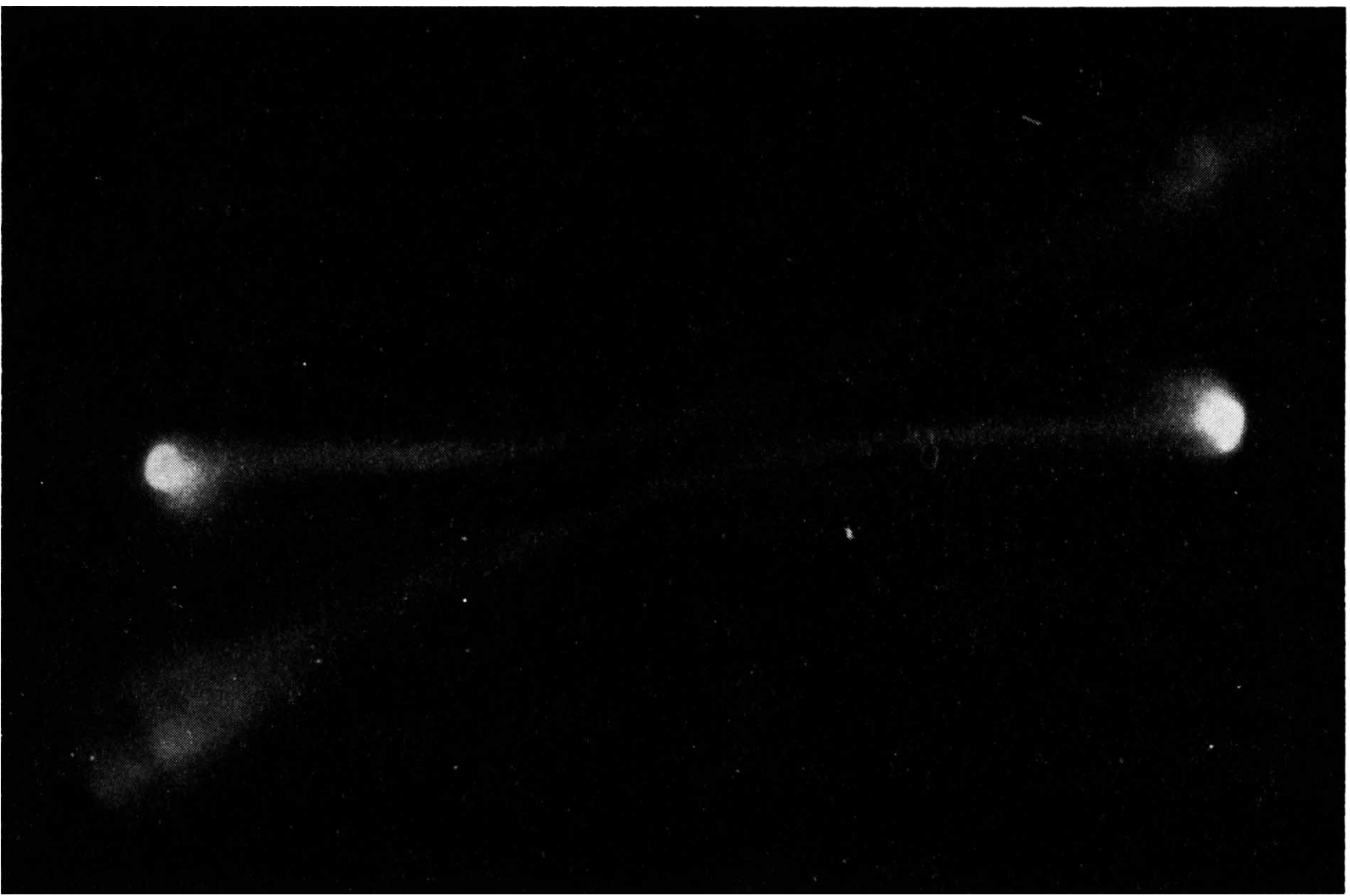

FIc. 1. Trajectories of plasmoids fired simultaneously at one another across a magnetic field of 4000 gauss at a pressure of $10^{-5} \mathrm{~mm} \mathrm{Hg}$ with a separation distance of $10 \mathrm{~cm}$

drift velocities remain the same in the laboratory as in the galaxy.

In the laboratory experiments, the Larmor radius will be much larger in proportion to the size of the phenomenon than it is in the galaxy. However, this disproportion should not upset the simulation procedure if, as in the present laboratory experiments, the Larmor radius of the electrons is small compared with the configuration being simulated. Furthermore, in both our experiments and in the galaxy, the gyrofrequency, $e H / m c$, for both positive ions and electrons exceeds the collision frequency. We thus believe it possible to simulate certain gross features of the galactic situation by the transformations indicated in Table I. In candor, we must point out that the interesting laboratory effects were discovered first, and that we now attempt an $a$ posteriori justification for using the discovered effects as simulations of galactic behavior.

The intrinsic power and beauty of the present simulation technique lies in the fact that the plasma can be photographed in three dimensions by its own recombination light. Thus, we can observe effects which are much more intricate than could be observed in plasmas with probes or microwave techniques. It is difficult to see how the effects we observe could ever be detected in liquid sodium or mercury.

When the plasma guns are fired across a magnetic field in a good vacuum $\left(10^{-6}-10^{-5} \mathrm{~mm} \mathrm{Hg}\right)$, they leave a luminous ${ }^{1,3}$ two-line "signature" or track which can be photographed. When two plasma guns are fired at one another, the photographed tracks (Fig. 1) show that the two pieces of plasma bounce off one another. Since the pieces of plasma retain their identity in this somewhat violent encounter, they are considered to be plasma-

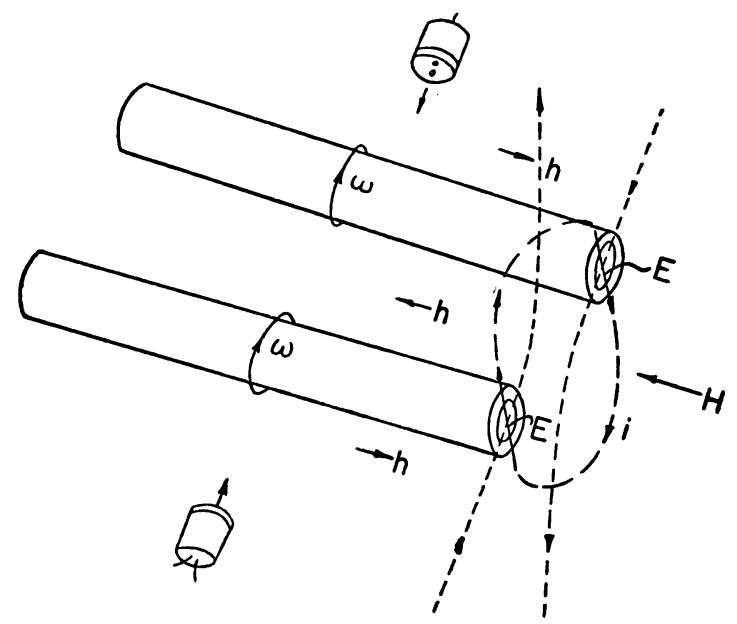

FIG. 2. Diagram of two plasmoids encountering each other as they travel across a magnetic field in a good vacuum, as in Fig. 1. 


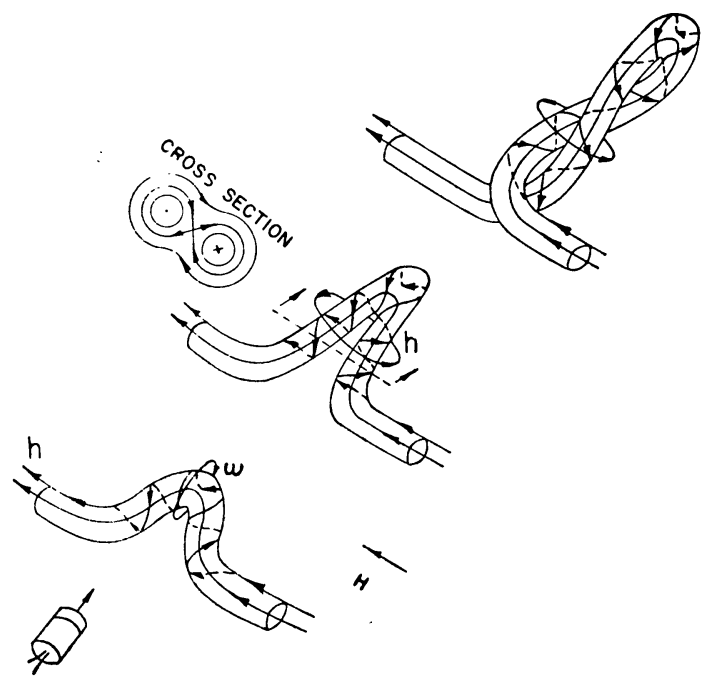

Fig. 3. Suggested configuration of plasma and magnetic field of a single plasmoid projected across a magnetic field when an electromagnetic braking action occurs because of a pressure of about $1 \mu$ in the vacuum chamber.

magnetic entities, and are accordingly called "plasmoids." A diagram of the shape of the plasmoids and the currents and magnetic fields generated by the encounter is given in Fig. 2. Probe measurements suggest that the plasmoid is a hollow cylinder; we hypothesize that it is a spin of the plasmoid which produces the hollow structure.

If a plasmoid is projected across a conducting medium (an ionized gas at $10^{-3} \mathrm{~mm} \mathrm{Hg}$ ), electromagnetic breaking action should trap the lines of magnetic field in the plasmoid. Accordingly, the dc magnetic-field lines will be dragged and twisted and the plasmoid shape will be distorted. Figure 3 illustrates the suggestion. Two such distorted plasmoids, fired at one another as diagramed in Fig. 4, may coalesce, because the juxtaposition of the apexes of the plasmoids could bring about stagnation points in the magnetic field, and the magnetic field lines could jump from one plasmoid to the other. Figure 5 illustrates distorted plasmoids seen to glue on to one another in a head-to-head encounter, while

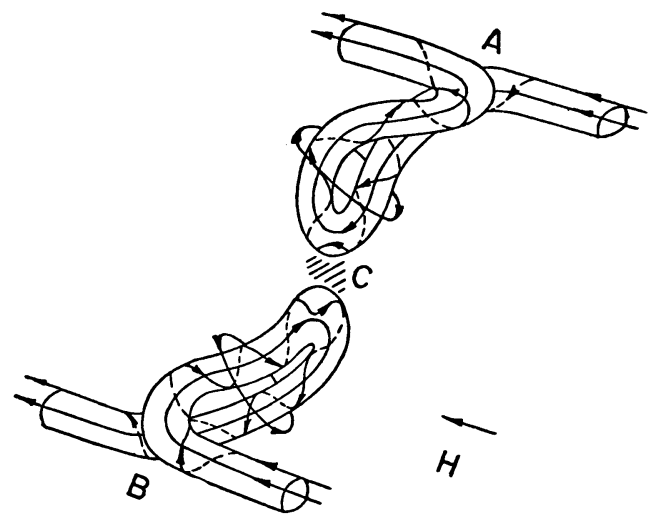

Fig. 4. Suggested encounter of two plasmoids which have been each twisted by braking action of ambient magnetic field.
Fig. 6 shows them to studiously repel one another on a flank-to-flank encounter.

It is possible to fire two plasmoids at one another and have them fasten head-to-tail to form a flattened ring which flips into a "figure 8."

It is also possible to fire four or eight plasmoids at one another and produce spirals which end up as rings, as shown in Fig. 7. The ring may remain stationary in the magnetic field, but in other cases the ring moves toward the camera.

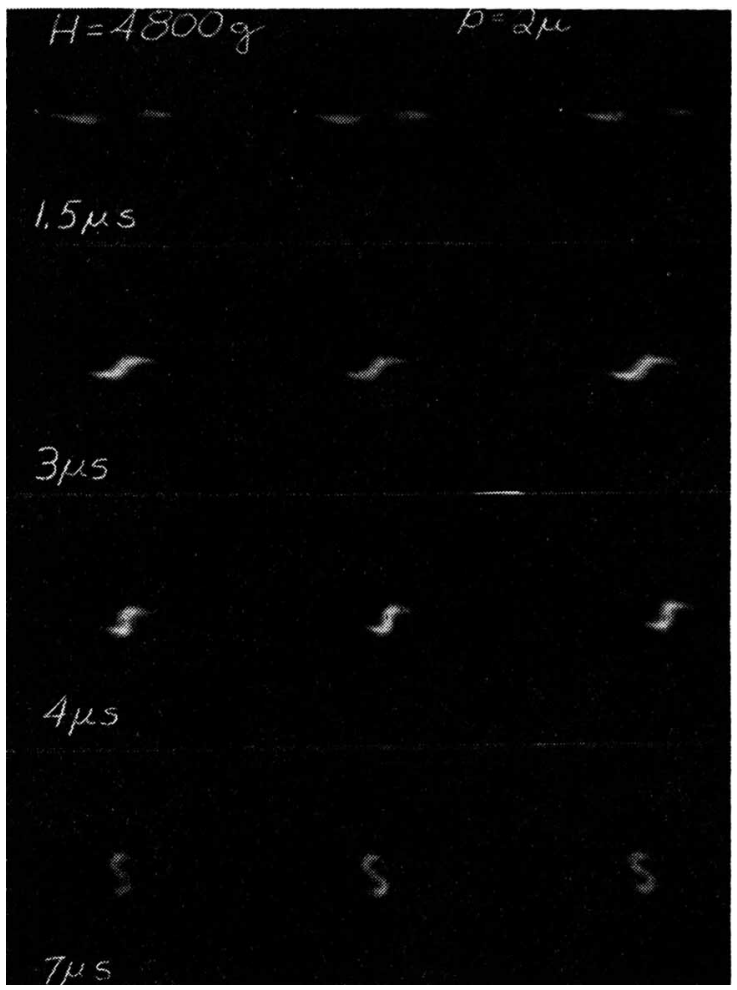

Frg. 5. A sequential study of two plasmoids fired from sources $10 \mathrm{~cm}$ apart across a magnetic field of 4800 gauss. The Kerr-cell exposure times are $2 \mu \mathrm{sec}$ and the various delay times of the sequence are indicated in $\mu \mathrm{sec}$. The pressure in the chamber is $2 \mu$. The plasmoids and their trajectories are rendered luminous primarily by the recombination light of the titanium and deuterium ions that come from the plasma source. The photographs on the left are the left stereoscopic photographs and those on the right, the right stereoscopic photographs, with an angle between the two views of $10^{\circ}$. The middle photograph is taken straight ahead along the direction of the magnetic field. The current (3000 amp for $0.4 \mathrm{sec}$ ) through the source produces a magnetic field which opposes the dc field and diminishes the velocity of projection of the plasmoid across the magnetic field.

Consider a completely ionized region of space $10^{25} \mathrm{~cm}$ in diameter. Statistical fluctuations in density will produce centers of incipient gravitational attraction in regions where the density is somewhat higher than in surrounding regions. As Jeans has shown, this process is essentially the amplification of a gravitational instability, and the matter will end up in clumps. The new ingredient we add to Jeans' picture is the magnetic field which might be generated by this process. We suggest that 
alignment of electron spins and cooperative interaction of electric currents carried by both electrons and positive ions may lead to an incipient magnetic field. It is difficult to show how this magnetic field could grow initially by regenerative dynamo action, although we can make out a fair case for the existence of dynamo action during a later phase of the galaxy.

We assume that the magnetic field of this region of higher density to a certain extent inhibits the motion of plasma across the magnetic field. However, the plasma will be free to travel along the lines of magnetic force; hence, by gravitational contraction the plasma can be expected to form the lenticular shaped concentration characteristic of a galaxy seen edge-on [see Fig. 8(a)]. Rayleigh-Taylor instability may now occur, with the plasma being supported against the central gravitational field by the magnetic field. The plasma may be expected to jet inward [see Fig. 8(b)] carrying, distorting, and twisting the lines of force as our plasmoids apparently do in the laboratory, as suggested by Figs. 1-7. Indeed

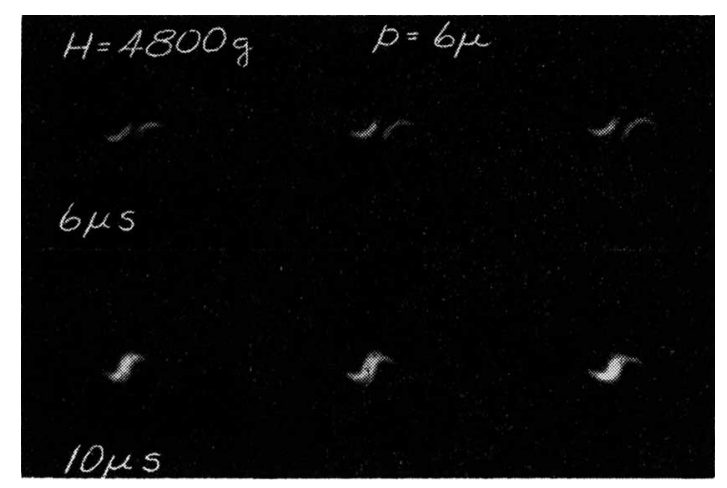

FIG. 6. The same as Fig. 5 except that the pressure in the chamber is $6 \mu$.

our laboratory experiments where we project plasma across a magnetic field are essentially a demonstration of this form of Rayleigh-Taylor instability since the magnetic field attempts to slow down the plasma which proceeds from the gun. As the plasma is initially decelerated by the magnetic field and by any subsequent electromagnetic breaking forces, it feels a deceleration in the direction in which it was projected; the plasma forms a jet which moves across the magnetic field (see Fig. 5).

We should expect these instability jets in the galaxy to spiral in toward the center [Fig. 8(b)] as they do in the laboratory experiments. Presumably if there are only two jets they will form a barred spiral galaxy (Figs. 5 and 6), but several jets will form a several armed spiral (Fig. 7). We thus draw the sequence of Figs. $8(\mathrm{a}), 8(\mathrm{~b}), 8(\mathrm{c})$, and $8(\mathrm{~d})$ which indicates our picture of galaxy formation. The new population type I stars are presumably condensed out of the hydrogen plasma which has been concentrated in the arms. These stars which get their nuclear energy from the $\mathrm{H}-\mathrm{H}$

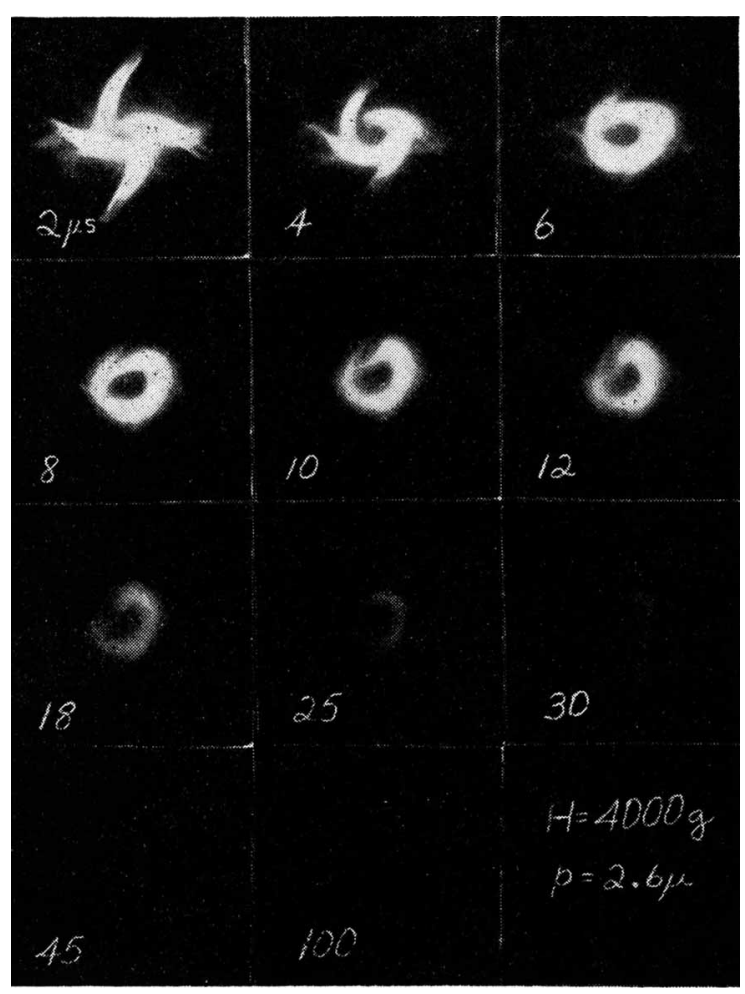

FIG. 7. An example of the formation of a ring by firing four sources across a magnetic field of 4000 gauss (into the paper) at a pressure of $2.6 \mu$. The exposure time is $2 \mu \mathrm{sec}$ and the various delay times are indicated in $\mu \mathrm{sec}$. The current in the sources is in such a direction that the velocity of projection of the plasma is retarded by the dc magnetic field. The $100-\mu$ sec delay photograph actually shows a faint ring on the original.

cycle are the stars which form the major portion of the population of these arms and thereby lend the arms their luminous structure. It can be seen from the diagrams of Figs. 3, 4, and 8 that the magnetic field of such a Taylor-instability jet which has become a spiral arm will be predominantly parallel to the arm. Chandrasekhar and $\mathrm{Fermi}^{6}$ have shown that a magnetic field of $10^{-6}$ gauss in the arms is necessary to prevent the complete gravitational collapse of the ionized hydrogen present in the arms.

We can now make plausible a magnetic-field and electric-current pattern which may lead to regenerative dynamo action in the galaxy, hence to a galactic magnetic field which grows in time as gravitational energy is transformed into magnetic energy, as shown in Fig. 8(d). The electromotive force which drives the currents from the periphery of the arms along the arms toward the center in the galaxy [Fig. 8(d)] is strictly analogous to the electromotive force which must exist in the laboratory barred spiral (Fig. 5) where the configuration can be observed to rotate in a magnetic field. The current configuration diagramed in Fig. 8(d) suggests a manner

6 S. Chandrasekhar and E. Fermi, Astrophys J. 118, 113, 116 (1953). T. G. Cowling, Magnetohydrodynamics (Interscience Publishers, Inc., New York, 1956), p. 6. 


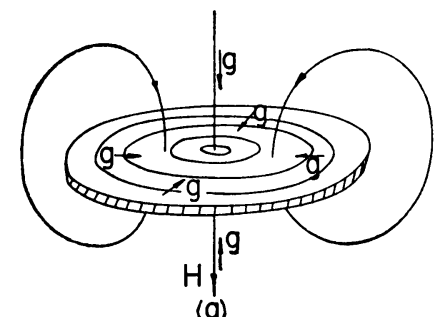

(a)

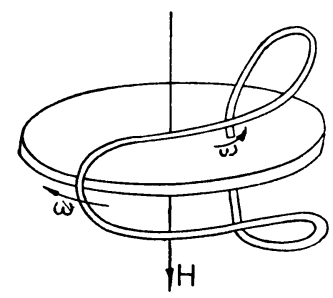

(C)

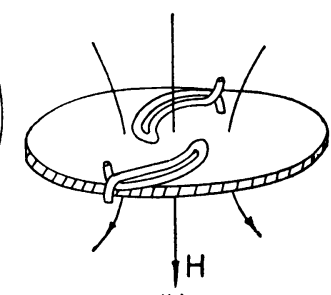

(b)

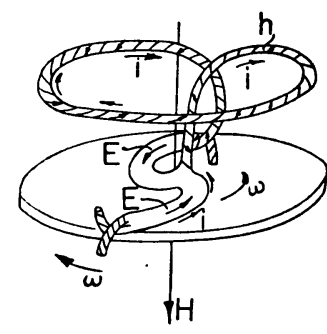

(d)
Fic. 8. Hypothesis on the process of formation of a barred spiral galaxy. (a) The pancake or lenticular shape produced by the gravitational attraction of a hydrogen plasma (protons and electrons) in conjunction with the magnetic field shown. (b) The origin of the arms produced by plasma jets moving across the magnetic field. The jets are presumably caused by Taylor instability. (c) A suggestion as to how a tube of magnetic flux of a dipole field will be dragged by the differential rotation in the galaxy. (d) A suggestion as to how regenerative dynamo action can occur in the galaxy to produce a galactic magnetic field which increases as time goes on. Electric current flow from galactic center to periphery via the galactic halo is indicated to show how the initial magnetic field $\mathrm{H}$ can be reinforced.

in which the dipole field of the galaxy can be reinforced by the currents which are driven along the arms and which return from the center to the periphery by flowing through the galactic halo.

If the general dipole field of the galaxy continues to rise in magnitude, any electrically conducting material (hydrogen plasma) in its neighborhood will have induced within it currents whose magnetic field will be repelled by the galaxy. If this hydrogen plasma represents an incipient galaxy in proximity to the other galaxy, it will be repelled by the latter and vice versa.

Indeed we can make a rough estimate of the velocity of recession which will be produced by this magnetic repulsion between galaxies. If the magnetic dipole field strength within the galaxy is about $5 \times 10^{-6}$ gauss, and the effective volume occupied by this field (for purposes of energy calculation) is about 25 times that of the galactic disk, the magnetic energy available per $\mathrm{cm}^{3}$ of the galactic disk for acceleration of the galaxy will be $25 \times\left(5 \times 10^{-6}\right)^{2} / 8 \pi \mathrm{ergs}=25 \times 10^{-12} \mathrm{erg}$. If the acceleration of one galaxy with respect to the other involves a mass corresponding to one hydrogen ion pair per $\mathrm{cm}^{3}$ in each galaxy, and if this magnetic energy for each galaxy is transformed to kinetic energy with respect to the center of mass of the two galaxies, the velocity of recession of each galaxy from the common center of mass is $6 \times 10^{6} \mathrm{~cm} / \mathrm{sec}$. The velocity of recession of one galaxy from the other is twice this speed or $100 \mathrm{~km} / \mathrm{sec}$. We emphasize that this estimate of speed probably represents a lower limit on the speed to be expected because, when the two galaxies are young and in close proximity, it is quite possible that the magnetic fields in the region between the centers of the two galaxies are compressed to values higher than $5 \times 10^{-6}$ gauss. Hence, there is a very real possibility that the kinetic energies of the galaxies with respect to one another are produced by magnetic repulsion. It is presumably this kinetic energy which produces the expansion of the universe, the galaxies on the periphery receding more rapidly than those close by. It is interesting to note that the high plasma speeds which we have been able to generate in the laboratory with the plasma gun have been produced essentially by the process of magnetic repulsion.

\section{DISCUSSION}

E. N. PARKER, Enrico Fermi Institute for Nuclear Studies, University of Chicago, Chicago, Illinois: We must remember the immense scale and not negligible conductivity of the galaxy. If you sweep a galactic arm across a magnetic field, the field will not penetrate significantly and you will get zero electric field in the interior. You do get a field in the laboratory. I think your explanation is all right there, but when you scale it up to the galactic size, you do not get emf's induced in the galactic arm.

W. H. BosTick, Stevens Institute of Technology, Hoboken, New Jersey: If there is only the galactic arm and no electrical conductivity in the galactic halo, it would take very little force to rotate the galactic arms. The magnetic field would crawl right across the galactic arm without any difficulty. The galactic arm would merely be polarized along its length from the galactic center to its tip. This sets up a homopolar generator with no brushes on it. Now, if you are willing to put the brushes on it, and if you say that with the brushes on the conductivity is too great, then I may have to back down somewhat.

I am in no position to evaluate the conductivity of the galactic halo, and I have no idea how long the conductivity path is. However, I still believe that it is possible to generate magnetic-field lines by dynamo action, even if the conductivity is high. 